\title{
THE SPUTTERING TEMPERATURE OF A COOLING CYLINDRICAL ROD WITHOUT AND WITH AN INSULATED CORE IN A TWO-FLUID MEDIUM
}

\author{
R. K. BERA ${ }^{1}$ and A. CHAKRABARTI ${ }^{2}$ \\ (Received 29 October 1993; revised 22 November 1994)
}

\begin{abstract}
Utilising Jones' method associated with the Wiener-Hopf technique, explicit solutions are obtained for the temperature distributions on the surface of a cylindrical rod without an insulated core as well as that inside a cylindrical rod with an insulated inner core when the rod, in either of the two cases, is allowed to enter, with a uniform speed, into two different layers of fluid with different cooling abilities. Simple expressions are derived for the values of the sputtering temperatures of the rod at the points of entry into the respective layers, assuming the upper layer of the fluid to be of finite depth and the lower of infinite extent. Both the problems are solved through a three-part Wiener-Hopf problem of special type and the numerical results under certain special circumstances are obtained and presented in tabular forms.
\end{abstract}

\section{Introduction}

The two physical problems (referred to as Problems I and II respectively) considered in this paper involve the determination of the temperature distributions on the surface of a cylindrical rod without an insulated core (I) and (II) inside the surface of a cylindrical rod with an insulated core; the heated rod is allowed to enter with a uniform speed $v$ a two-fluid medium of different depths with two different rates of cooling, producing two quench fronts (see [3]) that also propagate with the same speed. Recently, in the course of analysing the phenomena of cooling of a single infinite slab as well as a composite slab, in a two-fluid medium, Bera and Chakrabarti ([1], [2]) have generalised the mixed boundary-value problems considered in $[3,4,5,7,8]$ where similar cooling phenomena have been investigated for a single fluid medium.

The principal mathematical tool employed to solve such mixed boundary-value

\footnotetext{
${ }^{1}$ Department of Mathematics, Presidency College, BK-301, Salt Lake City, Calcutta- 700 091, India.

${ }^{2}$ Department of Applied Mathematics, Indian Institute of Science, Bangalore - 560012 , India.

(C) Australian Mathematical Society, 1996, Serial-fee code 0334-2700/96
} 
problems is the Wiener-Hopf technique, as described in [9] and [10] and utilized for the problems considered in $[1,2,4,5,7,8]$. Following the methods of Jones [9], the two problems under consideration are reduced to those of solving two WienerHopf type functional relations, known as "three-part Wiener-Hopf problems" (see Chakrabarti [6]). This three-part Wiener-Hopf problem is solved by employing an idea similar to that of Jones (see Jones [9] and Chakrabarti [6]) after reducing the problem to a system of algebraic equations involving two-infinite unknowns, whose solutions are completed in a standard manner. As in Evans [7] and Chakrabarti [4], we find that the sputtering temperatures, that is, the temperatures on the surface of the cylinder at the points of entry to the two layers of fluids, can be calculated by means of simple formulae involving the modified Bessel functions, the radius of the cylinder for the first problem and the outer and inner radii of the cylinder for the second problem, the speed $v$, and the diffusivity $\kappa$, along with the rates $h_{1}$ and $h_{2}$ of cooling. Certain typical values of the various parameters of the problems are considered here for the purpose of numerical evaluation of the sputtering temperatures.

\section{Formulation of the problems and their reduction to Weiner-Hopf equations}

Problem I : Taking $r=b$ as the radius of the cylinder without a core, and using cylindrical polar coordinates $(r, \theta, \hat{x})$, with $\hat{x}$-axis along the axis of the cylinder, the mathematical problem of determining the temperature $u^{(1)}(r, \hat{x}, t)$ inside the cylinder is that of solving the diffusion equation

$$
\frac{\partial u^{(1)}}{\partial t}=\kappa\left(\frac{\partial^{2} u^{(1)}}{\partial \hat{x}^{2}}+\frac{\partial^{2} u^{(1)}}{\partial r^{2}}+\frac{1}{r} \frac{\partial u^{(1)}}{\partial r}\right),
$$

in the region occupied by the non-insulated material of the cylindrical rod, $0 \leq r \leq b$, $-\infty<\hat{x}<\infty$, where $\kappa$ is the thermal diffusivity of the material.

Assuming that the rod gets cooled by allowing it to enter, with a uniform speed $v$ in the positive $\hat{x}$ direction, into two different layers of cold fluids, one of finite depth and the other of infinite extent, and using the coordinate transformation

$$
x=\hat{x}+v t,
$$

where $\hat{x}$ is fixed to the rod and $x$ is measured with respect to the fluid, we have that

$$
u^{(1)}(r, \hat{x}, t) \leftrightarrow u^{(1)}(r, x)
$$

with $\partial / \partial t=v \partial / \partial x$. Then (2.1) transforms into the new form

$$
2 s \frac{\partial u^{(1)}}{\partial x}=\frac{\partial^{2} u^{(1)}}{\partial r^{2}}+\frac{1}{r} \frac{\partial u^{(1)}}{\partial r}+\frac{\partial^{2} u^{(1)}}{\partial x^{2}}
$$


with $s=v / 2 \kappa$.

Problem II : Similarly taking $r=a$ and $r=b$ as the inner and outer radii of the cylinder and using cylindrical polar coordinates $(r, \theta, \hat{x})$, with the $\hat{x}$-axis as the axis of the cylinder, the mathematical problem of determining the temperature $u^{(2)}(r, \hat{x}, t)$ is that of solving the diffusion equation

$$
\frac{\partial u^{(2)}}{\partial t}=\kappa\left(\frac{\partial^{2} u^{(2)}}{\partial \hat{x}^{2}}+\frac{\partial^{2} u^{(2)}}{\partial r^{2}}+\frac{1}{r} \frac{\partial u^{(2)}}{\partial r}\right)
$$

in the region occupied by the non-insulated material of the cylindrical rod, $a<r<b$, $-\infty<\hat{x}<\infty$, where $\kappa$ is the thermal diffusivity of the material. (The insulated inner core occupies the region $0 \leq r \leq a$.)

Proceeding as in Problem I, we transform (2.3) to an equation which is exactly the same as (2.2). Setting

$$
u^{(j)}(r, x)=1-\phi^{(j)}(x, r) e^{s x}, \quad(j=1,2)
$$

both the problems under consideration can be reduced to that of solving the partial differential equation

$$
\left(\frac{\partial^{2}}{\partial r^{2}}+\frac{1}{r} \frac{\partial}{\partial r}+\frac{\partial^{2}}{\partial x^{2}}-s^{2}\right) \phi^{(j)}=0, \quad(j=1,2)
$$

in the respective regions

$$
(0 \leq r \leq b,-\infty<x<\infty), \quad \text { for Problem I }
$$

and

$$
(a<r<b,-\infty<x<\infty), \quad \text { for Problem II, }
$$

subject to the following boundary and infinity conditions for each problem. Problem I:
(i) $\frac{\partial \phi^{(1)}}{\partial r}=0$ at $r=b, x<0$,
(ii) $\frac{\partial \phi^{(1)}}{\partial r}+h_{1} \phi^{(1)}=h_{1} e^{-s x}$ at $r=b, 0 \leq x \leq 1$,
(iii) $\frac{\partial \phi^{(1)}}{\partial r}+h_{2} \phi^{(1)}=h_{2} e^{-s x}$ at $r=b, 1<x<\infty$,
(iv) $\phi^{(1)} \sim \mathrm{O}\left(e^{-s x}\right)$ as $x \rightarrow+\infty$,
(v) $\phi^{(1)} \sim \mathrm{O}\left(e^{s x}\right)$ as $x \rightarrow-\infty$, 
(vi) $\phi^{(1)}$ and $\frac{\partial \phi^{(1)}}{\partial r} \sim O(1)$ as $x \rightarrow 0$ and $x \rightarrow 1$, for uniqueness of the solution. Problem II:

(i) $\frac{\partial \phi^{(2)}}{\partial r}=0$ at $r=a,-\infty<x<\infty$,

(ii) $\frac{\partial \phi^{(2)}}{\partial r}=0$ at $r=b, x<0$,

(iii) $\frac{\partial \phi^{(2)}}{\partial r}+h_{1} \phi^{(2)}=h_{1} e^{-s x}$ at $r=b, 0<x<1$,

(iv) $\frac{\partial \phi^{(2)}}{\partial r}+h_{2} \phi^{(2)}=h_{2} e^{-s x}$ at $r=b, 1<x<\infty$,

(v) $\phi^{(1)} \sim \mathrm{O}\left(e^{-s x}\right)$ as $x \rightarrow+\infty$,

(vi) $\phi^{(1)} \sim \mathrm{O}\left(e^{s x}\right)$ as $x \rightarrow-\infty$,

(vii) $\phi^{(2)}$ and $\frac{\partial \phi^{(2)}}{\partial r} \sim O(1)$ as $x \rightarrow 0$ and $x \rightarrow 1$, for uniqueness of the solution.

We define the following Fourier transforms:

$$
\begin{aligned}
\Phi^{(j)}(\alpha, r) & \equiv \Phi_{-}^{(j)}(\alpha, r)+\Phi_{1}^{(j)}(\alpha, r)+\Phi_{+}^{(j)}(\alpha, r), \\
\Phi^{\prime(j)}(\alpha, r) & \equiv \Phi_{-}^{(j)}(\alpha, r)+\Phi_{1}^{(j)}(\alpha, r)+\Phi_{+}^{(j)}(\alpha, r),
\end{aligned}
$$

with

$$
\begin{aligned}
& \Phi_{-}^{(j)}(\alpha, r)=\int_{-\infty}^{0} \phi^{(j)}(x, r) e^{i \alpha x} d x \\
& \Phi_{1}^{(j)}(\alpha, r)=\int_{0}^{1} \phi^{(j)}(x, r) e^{i \alpha x} d x \\
& \Phi_{+}^{(j)}(\alpha, r)=\int_{1}^{\infty} \phi^{(j)}(x, r) e^{i \alpha x} d x \\
& \Phi_{-}^{(j)}(\alpha, r)=\int_{-\infty}^{0} \frac{\partial \phi^{(j)}}{\partial r} e^{i \alpha x} d x, \\
& \Phi_{1}^{(j)}(\alpha, r)=\int_{0}^{1} \frac{\partial \phi^{(j)}}{\partial r} e^{i \alpha x} d x, \\
& \Phi_{+}^{(j)}(\alpha, r)=\int_{1}^{\infty} \frac{\partial \phi^{(j)}}{\partial r} e^{i \alpha x} d x, \quad(j=1,2),
\end{aligned}
$$

and by using the conditions (iv), (v) and (vi) for Problem I and (v), (vi) and (vii) for Problem II deduce that $\Phi_{+}^{(j)}$ and $\Phi_{+}^{(j)},(j=1,2)$, are analytic functions of $\alpha$ in the upper half-plane $\operatorname{Im}(\alpha)>-s$ and $\Phi_{-}^{(j)}$ and $\Phi_{-}^{(j)},(j=1,2)$, are analytic in the 
lower half-plane $\operatorname{Im}(\alpha)<+s$ of the complex $\alpha$-plane, while $\Phi_{1}^{(j)}$ and $\Phi_{1}^{(j)}$ are entire functions of $\alpha$, so that $\Phi^{(j)}(\alpha, r)$ and $\Phi^{(j)}(\alpha, r),(j=1,2)$, are analytic in the strip $|\operatorname{Im}(\alpha)|<s$.

In the case of Problem I, the appropriate solution of (2.4) can be taken in the form

$$
\Phi(1)(\alpha, r)=C_{1} I_{0}(\gamma r)
$$

where $\gamma=+\left(\alpha^{2}+s^{2}\right)^{1 / 2}$, with the positive branch of the square root, $C_{1}$ is an arbitrary constant and $I_{0}(\gamma r)$ denotes the modified Bessel function of order zero. Applying the Fourier transform to the boundary conditions (i), (ii) and (iii) for Problem I along with the use of (2.7), we obtain the relations

$$
\begin{gathered}
\Phi^{\prime(1)}(\alpha, b) \equiv \Phi_{+}^{(1)}(\alpha, b)+\Phi_{1}^{(1)}(\alpha, b)=C_{1} \gamma I_{1}(\gamma, b) \\
\Phi_{1}^{(1)}(\alpha, b)+h_{1} \Phi_{1}^{(1)}(\alpha, b)=\frac{h_{1}}{s-i \alpha}\left[1-e^{-1(s-i \alpha)}\right] \\
\Phi_{+}^{(1)}(\alpha, b)+h_{2} \Phi_{+}^{(1)}(\alpha, b)=\frac{h_{2}}{s-i \alpha} \cdot e^{-1(s-i \alpha)} \\
C_{1}\left[\gamma I_{1}(\gamma b)+h_{1} I_{0}(\gamma b)\right]=h_{1} \Phi_{-}^{(1)}(\alpha, b)-\frac{\left(h_{1}-h_{2}\right)}{h_{2}} \cdot \Phi_{+}^{(1)}(\alpha, b)+\frac{h_{1}}{s-i \alpha} .
\end{gathered}
$$

Eliminating $C_{1}$ from (2.8) and (2.11), we obtain the following three-part Weiner-Hopf functional relation for the determination of the three unknown functions $\Phi_{-}^{(1)}(\alpha, b)$ $\left[\equiv \Phi_{-}^{(1)}(\alpha)\right], \Phi_{+}^{(1)}(\alpha, b)\left[\equiv \Phi_{+}^{(1)}(\alpha)\right]$, and $\Phi_{1}^{(1)}(\alpha, b)\left[\equiv \Phi_{1}^{(1)}(\alpha)\right]$, for the problem I:

$$
\Phi_{-}^{(1)}(\alpha)-\left(1 / h_{1}\right) K_{1}^{(1)}(\alpha) \Phi_{1}^{\prime(1)}(\alpha)-\left(1 / h_{2}\right) K_{2}^{(1)}(\alpha) \Phi_{+}^{(1)}(\alpha)=-1 /(s-i \alpha),
$$

valid in the strip $|\operatorname{Im}(\alpha)|<s$, where

$$
\begin{aligned}
& K_{1}^{(1)}(\alpha)=1+\frac{h_{1}}{\gamma} \cdot \frac{I_{0}(\gamma b)}{I_{1}(\gamma b)}, \\
& K_{2}^{(1)}(\alpha)=1+\frac{h_{2}}{\gamma} \cdot \frac{I_{0}(\gamma b)}{I_{1}(\gamma b)} .
\end{aligned}
$$

In the case of Problem II, the appropriate solution of the transformed equation (2.4) satisfying condition (i) can be taken in the form

$$
\Phi^{(2)}(\alpha, r)=C_{1}\left[I_{0}(\gamma r) K_{1}(\gamma a)+I_{1}(\gamma a) K_{0}(\gamma r)\right],
$$

where $\gamma=+\left(\alpha^{2}+s^{2}\right)^{1 / 2}$ as has been used earlier, $C_{1}$ is an artibrary constant and $I_{n}(X)$ and $K_{n}(x)$ denote modified Bessel functions of order $n$ of the first and second kinds respectively.

Proceeding as in Problem I and using the conditions (ii), (iii) and (iv) for the Problem II along with the use of (2.14) and finally eliminating the constant $C_{1}$ from 
the resulting equations, we obtain the following different three-part Wiener-Hopf functional relation for the determination of the three unknown functions $\Phi_{-}^{(2)}(\alpha)$, $\Phi_{+}^{\prime(2)}(\alpha)$ and $\Phi_{1}^{\prime(2)}(\alpha)$, for the Problem II :

$$
\Phi_{-}^{(2)}(\alpha)-\left(1 / h_{1}\right) K_{1}^{(2)}(\alpha) \Phi_{1}^{(2)}(\alpha)-\left(1 / h_{2}\right) K_{2}^{(2)}(\alpha) \Phi_{+}^{(2)}(\alpha)=-1 /(s-i \alpha),
$$

valid in the strip $|\operatorname{Im}(\alpha)|<s$, where

$$
\begin{aligned}
& K_{1}^{(2)}(\alpha)=1+\frac{h_{1}}{\gamma} \cdot \frac{I_{0}(\gamma b) K_{1}(\gamma a)+I_{1}(\gamma a) K_{0}(\gamma b)}{I_{1}(\gamma b) K_{1}(\gamma a)-I_{1}(\gamma a) K_{1}(\gamma b)}, \\
& K_{2}^{(2)}(\alpha)=1+\frac{h_{2}}{\gamma} \cdot \frac{I_{0}(\gamma b) K_{1}(\gamma a)+I_{1}(\gamma a) K_{0}(\gamma b)}{I_{1}(\gamma b) K_{1}(\gamma a)-I_{1}(\gamma a) K_{1}(\gamma b)} .
\end{aligned}
$$

If $h_{1}=h_{2}$ and $l=0$, we obtain that $K_{1}^{(2)}(\alpha)=K_{2}^{(2)}(\alpha)$; then (2.15) exactly coincides with (9) of Chakrabarti [4].

\section{Solution of the Wiener-Hopf equations}

Using the two relations of (2.13), we have for Problem I

$$
K_{1}^{(1)}(\alpha)=\frac{h_{1}}{h_{2}} K_{2}^{(1)}(\alpha)+h_{1}\left(\frac{1}{h_{1}}-\frac{1}{h_{2}}\right) .
$$

We then factorize $K_{1}^{(1)}(\alpha)$ in the form

$$
K_{1}^{(1)}(\alpha)=K_{1}^{(1)+}(\alpha) K_{1}^{(1)-}(\alpha),
$$

where $K_{1}^{(1)+}(\alpha)$ and $K_{1}^{(1)-}(\alpha)$ are analytic in the overlapping half-planes $\operatorname{Im}(\alpha)>\tau_{-}$ and $\operatorname{Im}(\alpha)<\tau_{+}$respectively (the details of which will be presented below) and rewrite (2.12) as

$$
\begin{aligned}
& \frac{\Phi_{-}^{(1)}(\alpha)}{K_{1}^{(1)-}(\alpha)}-\frac{1}{h_{2}} \cdot \frac{K_{2}^{(1)}(\alpha)}{K_{1}^{(1)-}(\alpha)} \cdot \Phi_{+}^{(1)}(\alpha)-\frac{1}{h_{1}} K_{1}^{(1)+}(\alpha) \Phi_{1}^{(1)}(\alpha) \\
& =-\frac{1}{K_{1}^{(1)-}(\alpha)(s-i \alpha)}
\end{aligned}
$$

We can also express (3.1), after using the factorization (3.2), in the form

$$
K_{1}^{(1)+}(\alpha)=\frac{h_{1}}{h_{2}} \cdot \frac{K_{2}^{(1)}(\alpha)}{K_{1}^{(1)-}(\alpha)}+h_{1}\left(\frac{1}{h_{1}}-\frac{1}{h_{2}}\right) \cdot \frac{1}{K_{1}^{(1)-}(\alpha)},
$$


giving

$$
\frac{1}{h_{2}} \cdot \frac{K_{2}^{(1)}(\alpha)}{K_{1}^{(1)-}(\alpha)}=\frac{1}{h_{1}} \cdot K_{1}^{(1)+}(\alpha)-\left(\frac{1}{h_{1}}-\frac{1}{h_{2}}\right) \cdot \frac{1}{K_{1}^{(1)-}(\alpha)} .
$$

Using (3.4) in (3.3), we obtain

$$
\begin{aligned}
& \frac{\Phi_{-}^{(1)}(\alpha)}{K_{1}^{(1)-}(\alpha)}-\frac{1}{h_{1}} K_{1}^{(1)+}(\alpha) \Phi_{+}^{(1)}(\alpha)+\left(\frac{1}{h_{1}}-\frac{1}{h_{2}}\right) \frac{\Phi_{+}^{(1)}(\alpha)}{K_{1}^{(1)-}(\alpha)}-\frac{1}{h_{1}} K_{1}^{(1)+}(\alpha) \Phi_{1}^{(1)}(\alpha) \\
& =-\frac{1}{K_{1}^{(1)-}(\alpha)} \cdot \frac{1}{(s-i \alpha)} .
\end{aligned}
$$

Now,

$$
\begin{aligned}
K_{1}^{(1)}(\alpha) & =\frac{\gamma I_{1}(\gamma b)+h_{1} I_{0}(\gamma b)}{\gamma I_{1}(\gamma b)} \\
& =A \prod_{n=1}^{\infty}\left[\frac{\alpha^{2}+\alpha_{n}^{(1) 2}}{\alpha^{2}+\beta_{n}^{(1) 2}}\right](\text { say }), \quad\left(\operatorname{Re} \alpha_{n}^{(1)}, \operatorname{Re} \beta_{n}^{(1)}>0\right)
\end{aligned}
$$

where $\pm i \alpha_{n}^{(1)}, \pm i \beta_{n}^{(1)}$ are the zeros of $\gamma I_{1}(\gamma b)+h_{1} I_{0}(\gamma b)$ and $\gamma I_{1}(\gamma b)$ respectively, and $A$ is a known constant.

We also observe that $\left|K_{1}^{(1)}(\alpha)\right| \rightarrow$ constant as $|\alpha| \rightarrow \infty$. Writing

$$
\begin{gathered}
K_{1}^{(1)+}(\alpha)=A^{1 / 2} \prod_{n=1}^{\infty}\left[\left(\alpha+i \alpha_{n}^{(1)}\right) /\left(\alpha+i \beta_{n}^{(1)}\right)\right] \\
\left(\left|K_{1}^{(1)+}(\alpha)\right| \rightarrow \text { constant, as }|\alpha| \rightarrow \infty\right),
\end{gathered}
$$

which is free from zeros and poles in the lower half-plane $\tau=\operatorname{Im}(\alpha)>$ $\max \left(-\alpha_{1}^{(1)},-\beta_{1}^{(1)}\right)=\tau_{-}$, say, and

$$
\begin{gathered}
K_{1}^{(1)-}(\alpha)=A^{1 / 2} \prod_{n=1}^{\infty}\left[\left(\alpha-i \alpha_{n}^{(1)}\right) /\left(\alpha-i \beta_{n}^{(1)}\right)\right] \\
\left(\left|K_{1}^{(1)-}(\alpha)\right| \rightarrow \text { constant, as }|\alpha| \rightarrow \infty\right),
\end{gathered}
$$

which is free from zeros and poles in the lower half-plane $\tau=\operatorname{Im}(\alpha)<\min \left(\alpha_{1}^{(1)}, \beta_{1}^{(1)}\right)$ $=\tau_{+}$, say, we observe that for $\operatorname{Im}(\alpha)<C$ and $\tau_{-}<C<\tau_{+}$,

$$
\begin{aligned}
\frac{\Phi_{+}^{(1)}(\alpha)}{K_{1}^{(1)}(\alpha)} & \equiv \frac{1}{2 \pi i} \int_{-\infty+i c}^{\infty+i c} \frac{\Phi_{+}^{(1)}(\omega) d \omega}{K_{1}^{(1)-}(\omega)(\omega-\alpha)} \\
& =\frac{1}{A^{1 / 2}} \sum_{j=1}^{\infty}\left[\frac{i\left(\alpha_{j}^{(1)}-\beta_{j}^{(1)}\right)}{\left(i \alpha_{j}^{(1)}-\alpha\right)}\right] \cdot \prod_{\substack{n=1 \\
n \neq j}}^{\infty}\left[\frac{\left(\alpha_{j}^{(1)}-\beta_{j}^{(1)}\right)}{\left(\alpha_{j}^{(1)}-\alpha_{n}^{(1)}\right)}\right] \cdot \Phi_{+}^{(1)}\left(i \alpha_{j}^{(1)}\right),
\end{aligned}
$$


which can be derived by closing the contour in the upper half-plane, by a large semicircular arc.

Then using (3.7) in (3.5) and splitting additively into - and + functions, we obtain, by employing Liouville's Theorem,

$$
\begin{gathered}
\frac{\Phi_{-}^{(1)}(\alpha)}{K_{1}^{(1)-}(\alpha)}-\left(\frac{1}{h_{1}}-\frac{1}{h_{2}}\right) \frac{1}{A^{1 / 2}} \sum_{j=1}^{\infty}\left[\frac{i\left(\alpha_{j}^{(1)}-\beta_{j}^{(1)}\right)}{\left(i \alpha_{j}^{(1)}-\alpha\right)}\right] \cdot \prod_{\substack{n=1 \\
n \neq j}}^{\infty}\left[\frac{\left(\alpha_{j}^{(1)}-\beta_{n}^{(1)}\right)}{\left(\alpha_{j}^{(1)}-\alpha_{n}^{(1)}\right)}\right] \cdot \Phi_{+}^{(1)}\left(i \alpha_{j}^{(1)}\right) \\
=-\frac{1}{(s-i \alpha)} \cdot\left[\frac{1}{K_{1}^{(1)-}(\alpha)}-\frac{1}{K_{1}^{(1)-}(-i s)}\right]
\end{gathered}
$$

where $\alpha \in$ lower half-plane $\tau<\tau_{+}$.

We again rewrite (2.12) as

$$
\Phi_{1}^{(1)}(\alpha)=\frac{h_{1}}{K_{1}^{(1)}(\alpha)} \cdot\left[\Phi_{-}^{(1)}(\alpha)+\frac{1}{s-i \alpha}\right]-\frac{h_{1}}{h_{2}} K^{(1)}(\alpha) \Phi_{+}^{\prime(1)}(\alpha)
$$

where

$$
K^{(1)}(\alpha)=\frac{K_{2}^{(1)}(\alpha)}{K_{1}^{(1)}(\alpha)}=\frac{\gamma I_{1}(\gamma b)+h_{2} I_{0}(\gamma b)}{\gamma I_{1}(\gamma b)+h_{1} I_{0}(\gamma b)}
$$

and use the factorization, $K^{(1)}(\alpha)=K^{(1)+}(\alpha) K^{(1)-}(\alpha)$, where

$$
K^{(1) \pm}(\alpha)=B^{1 / 2} \prod_{n=1}^{\infty}\left[\frac{\alpha \pm i \alpha_{n}^{(1)}}{\alpha \pm i \alpha_{n}^{(1)}}\right]
$$

and $\pm i \alpha_{n}^{(1)}$ and $\pm i \alpha_{n}^{(1)}$ are the zeros of $\gamma I_{1}(\gamma b)+h_{1,2} I_{0}(\gamma b)$ respectively, and $B$ is a known constant, different from the constant $A$, appearing earlier. (Note that $\left|K_{1}^{(1) \pm}(\alpha)\right| \rightarrow$ constant, as $|\alpha| \rightarrow \infty$ in the respective half planes.)

Dividing both sides of (3.9) by $K^{(1)-}(\alpha) \cdot e^{i \alpha l}$, we obtain the relation

$$
\begin{aligned}
\frac{\Phi_{1}^{(1)}(\alpha) e^{-i \alpha l}}{K^{(1)-}(\alpha)}= & -\frac{h_{1}}{h_{2}} K^{(1)+}(\alpha) \Phi_{+}^{(1)}(\alpha) e^{-i \alpha l} \\
& +\frac{h_{1} e^{-i \alpha l}}{K_{1}^{(1)}(\alpha) K^{(1)-}(\alpha)} \cdot\left[\Phi_{-}^{(1)}(\alpha)+\frac{1}{s-i \alpha}\right] .
\end{aligned}
$$


Observe that we can write (see Jones [9] and Noble [10])

$$
\begin{aligned}
& \frac{h_{1} e^{-i \alpha l}}{K_{1}^{(1)}(\alpha) K^{(1)-}(\alpha)}\left[\Phi_{-}^{(1)}(\alpha)+\frac{1}{s-i \alpha}\right] \\
& =Y_{+}+Y_{-} \\
& \equiv \frac{1}{2 \pi i} \int_{-\infty+i d}^{\infty+i d} \frac{h_{1} e^{-i \omega l}}{K_{1}^{(1)}(\omega) K^{(1)}(\omega)}\left[\Phi_{-}^{(1)}(\omega)+\frac{1}{s-i \alpha}\right] \frac{d \omega}{\omega-\alpha} \\
& \quad-\frac{1}{2 \pi i} \int_{-\infty+i d^{\prime}}^{\infty+i d^{\prime}} \frac{h_{1} e^{-i \omega l}}{K_{1}^{(1)}(\omega) K^{(1)}(\omega)}\left[\Phi_{-}^{(1)}(\omega)+\frac{1}{s-i \alpha}\right] \frac{d \omega}{\omega-\alpha}
\end{aligned}
$$

where $Y_{+}$is analytic in $\tau>d$ and $Y_{-}$is analytic in $\tau<d^{\prime}$, with $\tau_{-}<d<d^{\prime}<\tau_{+}$. Then from (3.12a) and (3.12b), by using Liouville's theorem with the conditions (iv), (v) and (vi), along with the Abelian theorem on Fourier Transforms, we obtain that

$$
\begin{aligned}
& \frac{h_{1}}{h_{2}} \cdot K^{(1)+}(\alpha) \Phi_{+}^{(1)}(\alpha) e^{-i \alpha l} \\
&=\frac{h_{1}}{(B A)^{1 / 2}} \cdot \frac{1}{2 \pi i} \int_{-\infty+i d}^{\infty+i d} \prod_{n=1}^{\infty}\left[\frac{\left(\omega+i \beta_{n}^{(1)}\right)}{\left(\omega+i \alpha_{n}^{(1)}\right)} \cdot \frac{\left(\omega-i \beta_{n}^{(1)}\right)}{\left(\omega-i \alpha_{n}^{(1)}\right)}\right] \cdot e^{-i \omega t} \\
& \cdot\left[\Phi_{-}^{(1)}(\omega)+\frac{1}{s-i \omega}\right] \cdot \frac{d \omega}{\omega-\alpha},
\end{aligned}
$$

where $\alpha \in$ the region above the line $(-\infty+i d, \infty+i d)$.

Evaluating the integral in (3.13), by closing the contour in the lower half-plane and using the residues at the poles, we get

$$
\begin{aligned}
& \frac{h_{1}}{h_{2}} \cdot K^{(1)+}(\alpha) \Phi_{+}^{(1)}(\alpha) e^{-i \alpha l} \frac{h_{1}}{(B A)^{1 / 2}} \sum_{j=1}^{\infty}\left\{\Phi_{-}^{(1)}\left(-i \alpha_{j}^{(1)}\right) e^{-\alpha_{j}^{(1)} 1} \cdot \prod_{n=1}^{\infty}\left[\frac{\left(\beta_{n}^{(1)}+\alpha_{j}^{(1)}\right)}{-\left(\alpha_{j}^{(1)}+\alpha_{n}^{(1)}\right)}\right] \cdot \frac{\left(\beta_{j}^{(1)}-\alpha_{j}^{(1)}\right)}{\left(-i \alpha_{j}^{(1)}-\alpha\right)}\right. \\
&\left.\cdot \prod_{\substack{n=1 \\
n \neq j}}^{\infty}\left[\frac{\left(\beta_{n}^{(1)}-\alpha_{j}^{(1)}\right)}{i\left(\alpha_{n}^{(1)}-\alpha_{j}^{(1)}\right)}\right]\right\} \\
&-\frac{h_{1}}{(B A)^{1 / 2}}\left\{\sum_{j=1}^{\infty} \prod_{n=1}^{\infty}\left[\frac{\left(\beta_{n}^{(1)}+\alpha_{j}^{(1)}\right)}{-i\left(\alpha_{j}^{(1)}+\alpha_{n}^{(1)}\right)}\right]\right. \\
& \prod_{\substack{n=1 \\
n \neq j}}^{\infty}\left[\frac{\left(\beta_{n}^{(1)}-\alpha_{j}^{(1)}\right)}{i\left(\alpha_{n}^{(1)}-\alpha_{j}^{(1)}\right)}\right] \frac{\left(\beta_{j}^{(1)}-\alpha_{j}^{(1)}\right) e^{-\alpha_{j}^{(1)} l}}{\left(s-\alpha_{j}^{(1)}\right)\left(-i \alpha_{j}^{(1)}-\alpha\right)} \\
&\left.\quad+\frac{i e^{-s l}}{(-i s-\alpha)} \cdot \prod_{n=1}^{\infty}\left[\frac{\left(\beta_{n}^{(1)}-s\right)}{i\left(\alpha_{n}^{(1)}-s\right)} \cdot \frac{\left(\beta_{n}^{(1)}+s\right)}{-i\left(\alpha_{n}^{(1)}+s\right)}\right]\right\}
\end{aligned}
$$


where $\alpha \in$ upper half-plane $\tau>\tau_{-}$.

Using $\alpha=-i \alpha_{r}^{(1)}$ in (3.8) and $\alpha=i \alpha_{r}^{(1)}$ in (3.14), we obtain the following twoinfinite system of algebraic equations for the two-infinite unknowns $\Phi_{-}^{(1)}\left(-i \alpha_{r}^{(1)}\right) \equiv x_{r}$ and $\Phi_{+}^{(1)}\left(i \alpha_{r}^{(1)}\right) \equiv y_{r}$ respectively :

$$
a_{r}^{(1)} x_{r}^{(1)}+\sum_{j=1}^{\infty} b_{r j}^{(1)} y_{j}^{(1)}=f_{r}^{(1)} \quad(r=1,2, \ldots)
$$

and

$$
c_{r}^{(1)} y_{r}^{(1)}+\sum_{j=1}^{\infty} d_{r j}^{(1)} x_{j}^{(1)}=g_{r}^{(1)} \quad(r=1,2, \ldots),
$$

where

$$
\begin{aligned}
a_{r}^{(1)}= & \prod_{n=1}^{\infty}\left[\frac{\alpha_{r}^{(1)}+\beta_{n}^{(1)}}{\alpha_{r}^{(1)}+\alpha_{n}^{(1)}}\right], \quad c_{r}^{(1)}=\prod_{n=1}^{\infty}\left[\frac{\alpha_{r}^{(1)}+\alpha_{n}^{(1)}}{\alpha_{r}^{(1)}+\alpha_{n}^{(1)}}\right] \cdot e^{\alpha_{r}^{(1)} l}, \\
b_{r j}^{(1)}= & -\left(\frac{1}{h_{1}}-\frac{1}{h_{2}}\right)\left[\frac{\alpha_{j}^{(1)}-\beta_{j}^{(1)}}{\alpha_{r}^{(1)}-\alpha_{j}^{(1)}}\right] \cdot \prod_{\substack{n=1 \\
n \neq j}}^{\infty}\left[\frac{\alpha_{j}^{(1)}-\beta_{n}^{(1)}}{\alpha_{j}^{(1)}-\alpha_{n}^{(1)}}\right], \\
d_{r j}^{(1)}= & -\frac{h_{2}}{A B}\left[\frac{\beta_{j}^{(1)}-\alpha_{j}^{(1)}}{\alpha_{j}^{(1)}+\alpha_{r}^{(1)}}\right] \cdot \prod_{n=1}^{\infty}\left[\frac{\beta_{n}^{(1)}+\alpha_{j}^{(1)}}{\alpha_{j}^{(1)}+\alpha_{n}^{(1)}}\right] \cdot \prod_{\substack{n=1 \\
n \neq j}}^{\infty}\left[\frac{\beta_{n}^{(1)}-\alpha_{j}^{(1)}}{\alpha_{n}^{(1)}-\alpha_{j}^{(1)}}\right] \cdot e^{-\alpha_{j}^{(1)} l}, \\
f_{r}^{(1)}= & -\left(\frac{1}{s-\alpha_{r}^{(1)}}\right)\left[\prod_{n=1}^{\infty}\left(\frac{\alpha_{r}^{(1)}+\beta_{n}^{(1)}}{\alpha_{r}^{(1)}+\alpha_{n}^{(1)}}\right)-\prod_{n=1}^{\infty}\left(\frac{s+\beta_{n}^{(1)}}{s+\alpha_{n}^{(1)}}\right)\right], \\
g_{r}^{(1)}= & \frac{h_{2}}{A B}\left[\sum_{j=1}^{\infty}\left(\frac{\beta_{j}^{(1)}-\alpha_{j}^{(1)}}{\alpha_{r}^{(1)}+\alpha_{j}^{(1)}}\right) \cdot \frac{e^{-\alpha_{j}^{(1)} l}}{\left(s-\alpha_{j}^{(1)}\right)} \cdot \prod_{n=1}^{\infty}\left(\frac{\beta_{n}^{(1)}+\alpha_{j}^{(1)}}{\alpha_{j}^{(1)}+\alpha_{n}^{(1)}}\right)\right. \\
& \left.\cdot \prod_{n=1}^{\infty}\left[\frac{\beta_{n}^{(1)}-\alpha_{j}^{(1)}}{\alpha_{n}^{(1)}-\alpha_{j}^{(1)}}\right]+\frac{e^{-s l}}{\left(s+\alpha_{r}^{(1)}\right)} \cdot \prod_{n=1}^{\infty}\left(\frac{\beta_{n}^{(1)}-s}{\alpha_{n}^{(1)}-s}\right) \cdot\left(\frac{\beta_{n}^{(1)}+s}{\alpha_{n}^{(1)}+s}\right)\right] .
\end{aligned}
$$

It is rather difficult to establish the uniqueness of the solution of the above very complex-looking system (3.15), but the fact that the boundary-value problems under consideration possess unique solutions may be convincing.

Proceeding exactly in the same manner as in Problem I and replacing $K_{1}^{(1)}(\alpha)$ and $K_{2}^{(1)}(\alpha)$ by $K_{1}^{(2)}(\alpha)$ and $K_{2}^{(2)}(\alpha) ; \Phi_{-}^{(1)}(\alpha), \Phi_{+}^{(1)}(\alpha)$ and $\Phi_{1}^{\prime(1)}(\alpha)$ by $\Phi_{-}^{(2)}(\alpha), \Phi_{+}^{(2)}(\alpha)$ and $\Phi_{1}^{(2)}(\alpha) ; K_{1}^{(1) \pm}(\alpha)$ and $K^{(1) \pm}(\alpha)$ by $K_{1}^{(2) \pm}(\alpha)$ and $K^{(2) \pm}(\alpha)$ respectively, we shall arrive at the system of equations similar to that of (3.15) for Problem II. The only other change is that $\pm i \alpha_{n}^{(2)}$ and $\pm i \beta_{n}^{(2)}$ are the zeros of

$$
\gamma\left[I_{1}(\gamma b) K_{1}(\gamma a)-I_{1}(\gamma a) K_{1}(\gamma b)\right]+h_{1}\left[I_{0}(\gamma b) K_{1}(\gamma a)+I_{1}(\gamma a) K_{0}(\gamma b)\right]
$$


and

$$
\gamma\left[I_{1}(\gamma b) K_{1}(\gamma a)-I_{1}(\gamma a) K_{1}(\gamma b)\right]
$$

respectively and $\pm i \alpha_{n}^{(2)}$ and $\pm i \alpha_{n}^{(2)}$ are the zeros of

$$
\gamma\left[I_{1}(\gamma b) K_{1}(\gamma a)-I_{1}(\gamma a) K_{1}(\gamma b)\right]+h_{1,2}\left[I_{0}(\gamma b) K_{1}(\gamma a)+I_{1}(\gamma a) K_{0}(\gamma b)\right]
$$

respectively.

It is interesting to note that the Wiener-Hopf functional relations (2.12) and (2.15) containing the three unknowns $\Phi_{-}^{(j)}(\alpha), \Phi_{+}^{(j)}(\alpha)$ and $\Phi_{1}^{(j)}(\alpha),(j=1,2)$, in each of the two problems considered here, are tackled in such an elegant way (similar to that already reported in [1] and [2]), that the determination of only two unknown functions $\Phi_{-}^{(j)}(\alpha)$ and $\Phi_{+}^{(j)}(\alpha),(j=1,2)$ in each case will solve the problem completely.

\section{Calculation of sputtering temperatures}

The temperature distribution for the Problem I can be determined by using the relations (3.8) and (3.14). The sputtering temperature at $x=0$, that is, at the entry to the first fluid stratum, is obtained from the relation (see Chakrabarti [4]),

$$
\begin{aligned}
1-\phi^{(1)}(0, b) \equiv u_{0}^{(1)}(b)=\lim _{|\alpha| \rightarrow \infty}\left[1-i \alpha \Phi_{-}^{(1)}(\alpha)\right] \\
\quad=\frac{1}{k_{1}^{(1)-}(-i s)}-\left(\frac{1}{h_{1}}-\frac{1}{h_{2}}\right) \sum_{j=1}^{\infty} y_{j}^{(1)}\left(\alpha_{j}^{(1)}-\beta_{j}^{(1)}\right) \prod_{\substack{n=1 \\
n \neq j}}^{\infty}\left[\frac{\alpha_{j}^{(1)}-\beta_{n}^{(1)}}{\alpha_{j}^{(1)}-\alpha_{n}^{(1)}}\right]
\end{aligned}
$$

In the special case $h_{1}=h_{2}$, the expression for the sputtering temperature at $x=0$ reduces to the same form as obtained by Evans [7] and Chakrabarti [4].

The sputtering temperature for this problem at the entry to the second fluid stratum, that is, at $x=l$, is given by

$$
u^{(1)}(l, b)=1-\phi^{(1)}(l, b) e^{s l}
$$

where

$$
\phi^{(1)}(l, b)=-\lim _{|\alpha| \rightarrow \infty}\left[i \alpha e^{-i \alpha l} \Phi_{+}^{(1)}(\alpha)\right]
$$


Using (3.14), we finally derive that

$$
\begin{aligned}
& u^{(1)}(l, b)=\frac{1}{A B}\left[\sum_{j=1}^{\infty}\left[\frac{\beta_{j}^{(1)}-\alpha_{j}^{(1)}}{s-\alpha_{j}^{(1)}}\right] e^{-\alpha_{j}^{(1)} l} \prod_{n=1}^{\infty}\left(\frac{\beta_{n}^{(1)}+\alpha_{j}^{(1)}}{\alpha_{n}^{(1)}+\alpha_{j}^{(1)}}\right) \prod_{\substack{n=1 \\
n \neq j}}^{\infty}\left(\frac{\beta_{n}^{(1)}-\alpha_{j}^{(1)}}{\alpha_{n}^{(1)}-\alpha_{j}^{(1)}}\right)\right. \\
&+\left.\prod_{n=1}^{\infty}\left\{\left(\frac{\beta_{n}^{(1)}+s}{\alpha_{n}^{(1)}+s}\right)\left(\frac{\beta_{n}^{(1)}-s}{\alpha_{n}^{((1)}-s}\right)\right\} e^{-s l}\right] e^{s l} \\
&+\frac{1}{A B}[ {\left[\sum_{j=1}^{\infty} x_{j}^{(1)}\left(\beta_{j}^{(1)}-\alpha_{j}^{(1)}\right) e^{\left(s-\alpha_{j}^{(1)}\right) l}\right.} \\
&\left.\prod_{n=1}^{\infty}\left(\frac{\beta_{n}^{(1)}+\alpha_{j}^{(1)}}{\alpha_{n}^{(1)}+\alpha_{j}^{(1)}}\right) \prod_{\substack{n=1 \\
n \neq j}}^{\infty}\left(\frac{\beta_{n}^{(1)}-\alpha_{j}^{(1)}}{\alpha_{n}^{(1)}-\alpha_{j}^{(1)}}\right)\right]
\end{aligned}
$$

The temperature distribution for the Problem II can be determined in the similar way, and the sputtering temperatures at $x=0$ and at $x=l$ are respectively given by the relations

$$
u_{0}^{(2)}(b)=\frac{1}{K_{1}^{(2)-}(-i s)}-\left(\frac{1}{h_{1}}-\frac{1}{h_{2}}\right) \sum_{j=1}^{\infty} y_{j}^{(2)}\left(\alpha_{j}^{(2)}-\beta_{j}^{(2)}\right) \prod_{\substack{n=1 \\ n \neq j}}^{\infty}\left(\frac{\alpha_{j}^{(2)}-\beta_{n}^{(2)}}{\alpha_{j}^{(2)}-\alpha_{n}^{(2)}}\right)
$$

and

$$
\begin{gathered}
u^{(2)}(l, b)=\frac{1}{A B}\left[\sum_{j=1}^{\infty} \prod_{n=1}^{\infty}\left(\frac{\beta_{n}^{(2)}+\alpha_{j}^{(2)}}{\alpha_{j}^{(2)}+\alpha_{n}^{(2)}}\right) \prod_{\substack{n=1 \\
n \neq j}}^{\infty}\left(\frac{\beta_{n}^{(2)}-\alpha_{j}^{(2)}}{\alpha_{n}^{(2)}-\alpha_{j}^{(2)}}\right) \cdot\left(\frac{\beta_{j}^{(2)}-\alpha_{j}^{(2)}}{s-\alpha_{j}^{(2)}}\right)\right. \\
\left.\cdot e^{\left(s-\alpha_{j}^{(2)}\right) l}+\prod_{n=1}^{\infty}\left(\frac{\beta_{n}^{(2)}-s}{\alpha_{n}^{(2)}-s}\right)\left(\frac{\beta_{n}^{(2)}+s}{\alpha_{n}^{(2)}+s}\right)\right] \\
+\frac{1}{A B}\left[\sum_{j=1}^{\infty} x_{j}^{(2)} e^{\left(s-\alpha_{j}^{(2)}\right) l} \prod_{n=1}^{\infty}\left(\frac{\beta_{n}^{(2)}+\alpha_{j}^{(2)}}{\alpha_{j}^{(2)}+\alpha_{n}^{(2)}}\right)\right. \\
\left.\cdot\left(\beta_{j}^{(2)}-\alpha_{j}^{(2)}\right) \cdot \prod_{\substack{n=1 \\
n \neq j}}^{\infty}\left(\frac{\beta_{n}^{(2)}-\alpha_{j}^{(2)}}{\alpha_{n}^{(2)}-\alpha_{j}^{(2)}}\right)\right] .
\end{gathered}
$$

\section{Numerical results}

Numerical results are presented below in Tables 1 and 2 respectively for the sputtering temperatures $u_{0}^{(1)}(b)$ and $u^{(1)}(l, b)$ for Problem I and for $u_{0}^{(1)}(b)$ and $u^{(2)}(l, b)$ for Problem II at the points of entry to the first and second fluid strata for particular 
choices of $\lambda, \mu, s, l$ and $b$. Here $\lambda=b h_{1}$ and $\mu=b h_{2}$ with $b=1$ for Problem I and for other choices of $\lambda, \mu, \nu, s$, and $l$, where $\lambda=b h_{1}, \mu=b h_{2}, \nu=a / b$, for Problem II with $A B=1$. The zeros of $\gamma I_{1}(r b)+h_{1,2} I_{0}(\gamma b)$ are determined by the method of 'bisection' and are found to be correct up to seven decimal places. The zeros of $\gamma I_{1}(\gamma b)$ are available from standard books of tables of functions. Utilizing these roots, the system (3.15) is solved by the method of 'Gauss Elimination with Partial Pivoting'. These solutions and the roots already obtained are then used in (4.1), (4.4), (4.5) and (4.6) to determine the sputtering temperatures displayed in Tables 1 and 2. All these calculations were done on a PC. The method of calculation clearly ensures the correctness of the results to three significant figures which will be sufficient for such problems.

TABLE 1. Calculation of sputtering temperatures $u_{0}^{(1)}(b)$ and $u^{(1)}(l, b)$

\begin{tabular}{|c|c|c|c|c|c|c|}
\hline$\mu=.02$ & $\lambda=.04$ & $\lambda=.06$ & $\lambda=.08$ & $\lambda=.60$ & $\lambda=.80$ & $\lambda=1.0$ \\
$l=.02$ & $s=.10$ & $s=.10$ & $s=.10$ & $s=.105$ & $s=.105$ & $s=.105$ \\
\hline$u_{0}^{(1)}(b)$ & 0.359 & 0.405 & 0.449 & 0.551 & 0.653 & 0.747 \\
\hline$u^{(1)}(l, b)$ & 0.302 & 0.290 & 0.295 & 0.161 & 0.159 & 0.157 \\
\hline
\end{tabular}

TABLE 2. Calculation of sputtering temperatures $u_{0}^{(2)}(b)$ and $u^{(2)}(l, b)$

\begin{tabular}{|rc|c|c|c|c|c|c|}
\hline$\mu=.02$ & $\lambda=.04$ & $\lambda=.06$ & $\lambda=.08$ & $\lambda=.60$ & $\lambda=.80$ & $\lambda=1.0$ \\
& $l=.02$ & $s=.10$ & $s=.10$ & $s=.10$ & $s=.105$ & $s=.105$ & $s=.105$ \\
\hline \multirow{2}{*}{$\nu=0.1$} & $u_{0}^{(1)}(b)$ & 0.365 & 0.418 & 0.472 & 0.770 & 0.516 & 0.651 \\
& $u^{(2)}(l, b)$ & 0.304 & 0.295 & 0.291 & 0.289 & 0.181 & 0.177 \\
\hline \multirow{2}{*}{$\nu=0.2$} & $u_{0}^{(2)}(b)$ & 0.385 & 0.454 & 0.523 & 0.878 & 0.592 & 0.938 \\
& $u^{(1)}(l, b)$ & 0.310 & 0.306 & 0.306 & 0.313 & 0.145 & 0.220 \\
\hline
\end{tabular}

\section{Discussion}

The numerical results presented in Tables 1 and 2 for the two problems under consideration show clearly that the sputtering temperatures, at the two entry points, remain less than 1 , as expected. This fact establishes the power of the WienerHopf technique. Furthermore, it handles more complicated boundary-value problems associated with the diffusion equation than those handled previously. 
Some asymptotic results for large values of the depth of the upper fluid are derivable from the solutions obtained here. Because of the rather complex-looking expressions involved, such asymptotic calculations are deferred to a later work.

\section{Acknowledgements}

We are grateful to the referees for their valuable suggestions.

\section{References}

[1] R. K. Bera and A. Chakrabarti, "Cooling of a composite slab in a two-fluid medium", J. Appl. Math. and Phys. (ZAMP) 42 (1991) 943-959.

[2] R. K. Bera and A. Chakrabarti, "Cooling of an infinite slab in a two-fluid medium", J. Austral. Math. Soc. Ser. B 33 (1992) 474-485.

[3] R. E. Caflisch and J. B. Keller, "Quench front propagation", Nuclear Eng. and Design 65 (1981) 97-102.

[4] A. Chakrabarti, "The sputtering temperature of a cooling cylindrical rod with an insulated core", Appl. Sci. Res. 43 (1986) 107-113.

[5] A. Chakrabarti, "Cooling of a composite slab", Appl. Sci. Res. 43 (1986) 213-225.

[6] A. Chakrabarti, "A simplified approach to a three-part Wiener-Hopf problem arising in diffraction theory”, Math. Proc. Camb. Phil. Soc. 102 (1987) 371.

[7] D. V. Evans, "A note on the cooling of a cylinder entering a fluid", IMA J. Appl. Math. 33 (1984) 49-54.

[8] H. Hevine, "On a mixed boundary value problem of diffusion type", Appl. Sci. Res. 39 (1982) 261-276.

[9] D. S. Jones, Electromagnetic theory (Pergamon, London, 1964).

[10] B. Noble, Methods based on the Wiener-Hopf technique (Pergamon, London, 1958). 